\title{
Compte rendu de Degei's Descendants. Spirits, Place and People in Pre-Cession Fiji, de Matthew Spriggs et Deryck Scarr (eds)
}

\section{Gilles Bounoure}

\section{OpenEdition}

Édition électronique

URL : http://journals.openedition.org/jso/7400

DOI : $10.4000 /$ jso. 7400

ISSN : $1760-7256$

Éditeur

Société des océanistes

Édition imprimée

Date de publication : 15 décembre 2015

Pagination : 353-355

ISBN : 978-2-85430-126-7

ISSN : 0300-953x

Référence électronique

Gilles Bounoure, «Compte rendu de Degei's Descendants. Spirits, Place and People in Pre-Cession Fiji, de Matthew Spriggs et Deryck Scarr (eds) », Journal de la Société des Océanistes [En ligne], 141 | juilletdécembre 2015, mis en ligne le 03 janvier 2016, consulté le 24 septembre 2020. URL : http:// journals.openedition.org/jso/7400 ; DOI : https://doi.org/10.4000/jso.7400 
auteur et de ses éditeurs, l'histoire de ce livre ne présume évidemment ni de son utilité ni de son actualité, l'une et l'autre paraissant néanmoins hors de cause à lire les premiers mots de Parke, en tête de sa préface (XIX) :

"The first aim of my research is to determine, from oral accounts I recorded over a period of some fifty years, how Fijians especially in western areas of Fiji currently understand and explain (a) the origins, characteristics, development and interactions of the social and political divisions of late pre-Colonial traditional Fijian society, and (b) the general principles of traditional land tenure. The second aim is to assess the reasoning, consistency and, where possible, the historical accuracy of such understandings. "

Cette sorte de recherche n'a rien à redouter des résultats "positifs" mais toujours révisables de l'archéologie de terrain, et elle serait plutôt à même d'en orienter les intérêts, voire de lui désigner les secteurs à fouiller. Ainsi qu'écrit Parke (p. 16),

"Indeed it may have to be admitted that neither the NLC [Native Land Commission] accounts nor my oral accounts will ever be accepted as being historically accurate. The results of archaeological field surveys and linguistic investigations, however, should provide data from these other disciplines which throw light on the question of the accuracy or reasonableness of the recorded and oral accounts. »

Mais ce n'est pas si simple : il mentionne des vestiges ou des endroits remarquables par dizaines, tout en précisant qu'on ne visite pas ces lieux sacrés sans négociations ou cérémonies préalables, tant ils restent chargés de forces obscures à apprivoiser et de revendications foncières à continuer de faire valoir. Avant d'autres, Parke avait compris que c'était autrement plus difficile que d'exhumer des habitats celtes dans le Dorset ou des cimetières mérovingiens dans le centre de Paris.

Même s'il n'est pas formulé explicitement, le point de départ de ce long travail d'enquête se devine dans les premiers chapitres du livre : jeune cadre ("Officer») de l'administration coloniale britannique, l'auteur avait eu peine à démêler la situation - juridique, pratique, sociale - $\mathrm{du}$ « foncier » dans les districts dont on l'avait chargé. Cela remontait, il en démêle l'histoire, aux premiers actes d'Arthur Gordon, le gouverneur nommé après la cession de l'archipel à la Couronne britannique (1874), administration qualifiée tantôt de "généreuse " avec les autochtones, tantôt de "paresseuse " ou de "féodale ", mais essentiellement dépourvue de moyens et réduite à la vieille solution romaine : une "provincia" ne doit rien coûter à la métropole, c'est plutôt à l' "Urbs» que devraient en revenir les possibles revenus, et aux colonisés de s'arranger entre eux pour les fournir. Fut ainsi enregistrée une première cartographie des "land tenures", corrigée à maintes reprises jusqu'à une ultime réforme en 1945, celle qu'eut à faire respecter Parke et dont il vérifia rapidement tous les aspects flous ou filous, dans l'ouest et le nord-ouest de Viti Levu, les régions les moins précisément étudiées de la grande île, où se concentrent aussi les sites ou les projets miniers (or et cuivre, $c f$. Geopacific Resources, 2005, 2012; magnétite, $c f$. Amex, 2015). Parke n’y fait allusion qu'une seule fois (p. 204), à propos de sa visite d'un site mégalithique, "defaced by miners exploring the area known as Vatutu", riche en grès aurifère. Tels sont aussi les districts sur lesquels il a orienté sa recherche, déjà précieuse à ce seul titre.

C'est pourtant à proximité de la côte Est que les relations occidentales les plus anciennes situent le séjour du dieu ou héros civilisateur Degei :

"According to the universal belief of the natives, the supreme deity, and governor of their island-world, is a being termed Ndengei. He is represented as having the form of a serpent in the head and one side of the body, while the rest is made of stone, by which he is rendered immortal. His residence is in a cave, in the mountains of Viti-levu, at a place called Nakauvandra, nearly opposite to Mbua, or Sandalwood Bay"

écrit ainsi Hale (1846 : 52, non cité par Parke), même si son supérieur Wilkes (1845, III : 83) situe le lieu "near the western end of Vitilevu", sans doute par lapsus. Ajoutant ses précisions à celles du missionnaire Fison (voir aussi 1904 : 27-31, non utilisé par Parke), Joske (1889 : 266, cité par Parke p. 63 mais absent de sa bibliographie) indique :

"In some dialects $n$ Galongalo is synonomous with Kauvandra. The Kauvandra [a mountain range in the North of Vitilevu], is the home of nDegei (the Fijian Creator) and his cave, which I have visited, faces the N. W. "

Parmi les nombreuses pages qu'il lui consacre, Thomson $(1908: 112,134)$ le présente en ces termes :

"Ndengei, primarily a god of Rakiraki on the northeast coast of Vitilevu, but known throughout Fiji except in the eastern islands of the Lau group"

avant de suggérer son importance par cette observation :

"When the missionaries first attempted the conversion of Rakiraki the people thought that Christianity was a mere variant of their own cult of Ndengei, using the following argument: $N$ dengei $=$ the True God; Jehovah $=$ the True God; therefore, Jehovah = Ndengei. "

Identification encore facilitée par les légendes épiques lui attribuant la responsabilité d'un vaste déluge, déclenché pour châtier les meurtriers du pigeon sacré accoutumé à venir le réveiller. $\mathrm{Ce}$ qui a conduit des spécialistes à mettre en doute, à l'instar de Peter France (1966: 112, cité par Parke, p. 133), la valeur de ces légendes «born of 
missionary parentage, and nurtured by the enquiries of the Native Lands Commission."

Tout en admettant que les mythes associés à Degei furent "largely missionary-and anthropologistinfluenced", Parke soutient que pourtant "there could well be an original kernel, even if overlaid by accretions" (p. 29). La mise au jour de ce noyau primitif, qui constitue une autre grande ambition de l'ouvrage, repose évidemment sur l'apport d'informations inédites issues de la tradition orale, mais aussi sur une critique rigoureuse des sources, à nettoyer de leurs "accrétions". Ici réside la principale faiblesse de ce volume, qui fourmille de données et d'observations pour la plupart de première main, mais sans les références qui permettraient non pas de les authentifier, la sincérité de cette recherche étant éclatante, mais de les approfondir ou de les prolonger. C'est par exception que sont précisés le nom des informateurs et les circonstances dans lesquelles furent recueillis leurs propos, à des décennies de distance (1953: 113 et 235, ici sans nom d'informateur, 1995: 133). Plus fréquentes, les allusions vagues ("some said... others said...", p. 25, 29, etc., "some myths... other myths", p. 67) font regretter l'absence de tout appareil de notes, les éditeurs n'ayant pu mieux faire, après la destruction des vastes archives de Parke peu après son décès (M. Spriggs, p.IX), que de préciser les sources qui leur étaient connues. Voilà qui conduit aussi à recommander la version électronique proposée gratuitement par l'ANu E-Press (ISBN : 9781925021844) plutôt que la version imprimée (ISBN : 9781925021813), dépourvue d'index.

Mais ces éditeurs n'auront pas travaillé en vain, et il ne faudrait pas que ce livre, parce qu'il est inachevé, parfois un peu brouillon et répétitif comme le sont souvent les thèses, soit dédaigné à l'exemple des précédents travaux de Parke, assimilés à d'aimables divertissements d'" amateur enthousiaste". À côté d'informations orales qui mériteront certainement de longues enquêtes de la part des Fidjiens eux-mêmes, il rassemble des données historiques peu connues et utilisables sans délai (par exemple, pp. 45-56 sur l'établissement tardif des Blancs dans l'ouest de Viti Levu, la cession de terres par les chefs en échange de fusils, l'iconoclasme des missionnaires wesleyens, etc.), et il met surtout en évidence la fluidité des pratiques foncières, mais aussi sociales et religieuses prévalant avant que l'administration coloniale n'entreprenne de les figer, pour des résultats aussi désastreux aux Fidji que dans le reste du Pacifique.

\section{RÉFÉRENCES CITÉES}

Amex, 2015. Mba Delta Ironsands Viti Levu, Fiji (http://www.amex.net.au/projects/mbadelta-ironsands).
Clark Geoffrey and Atholl Anderson (eds), 2009. The early prehistory of Fiji, Canberra, Australian University Press.

Davidson Janet, 2008. The Archaeology of the Polynesian Homeland: A retrospective view of the early years, in David J. Addison and Christophe Sand, Recent Advances in the Archaeology of the Fiji/West-Polynesia Region, Dunedin, University of Otago Studies in Prehistoric Anthropology 21, pp. 7-31.

FIsON Lorimer, 1904. Tales from old Fiji, London, Alexander Moring.

France Peter 1966. The Kaunitoni Migration: Notes on the Genesis of a Fijian Tradition, Journal of Pacific History 1, pp. 107-113.

Geopacific Resources, 2005. (http://globaldocuments.morningstar.com/documentlibrary/document/e125b82582511a05.msdoc/original).

—, 2012. (http://geopacific.com.au/fiji-sabetovuda/) et (http://geopacific.com.au/fiji-nabila/).

Hale Horatio, 1846. United States Exploring Expedition... Ethnology and Philology, Philadelphia, Sherman.

Joske Adolph B., 1889. The Nanga of Viti Levu, Internationales Archiv für Ethnographie 2, pp. 254-271.

Thomson Basil, 1908. The Fijians. A study of the decay of custom, London, Heinemann.

Wilkes Charles, 1845. Narrative of the United States Exploring Expedition... III, Philadelphia, Lea \& Blanchard.

\section{Gilles Bounoure}

TABAni Marc and Marcellin ABong (eds), 2013. Kago, Kastom and Kalja: The Study of Indigenous Movements in Melanesia Today, Marseille, pacific-credo Publications, coll. Cahiers du CREDo, 218 p., bibliogr., 16 ill.

L'ouvrage, suite à deux sessions ASAO en 2009 et 2010, se propose de faire le point sur le concept, assez imprécis, de " cargo cult " - dont on a dit qu'il n'était ni cargo, ni culte et que plusieurs auteurs proposent de mettre entre guillemets inversés ou même

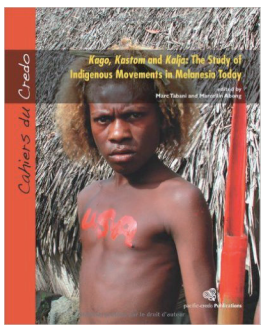
de l'écrire en le barrant -, et sur les tendances récentes de ces "mouvements" (nous les appelons ainsi pour rester "neutres ») après plusieurs décennies d'indépendance et de modernisation (Tabani). 